\title{
The effect of chlorhexidine in reducing oral colonisation in geriatric patients: a randomised controlled trial
}

\author{
Sharifah Shafinaz Binti Sharif-Abdullah ${ }^{1,2}$, MNSc, BNSc, Mei Chan $\underline{C h o n g}^{1}$, Phd, MSC, \\ Surat Singh Surindar-Kaur ${ }^{1}$, MNSc, BNSc, Shahrul Bahyah Kamaruzzaman ${ }^{3}$, PhD, MRCP, Kwan Hoong Ng ${ }^{4}$, PhD, DABMP
}

\begin{abstract}
INTRODUCTION Inadequate oral care has been implicated in the development of aspiration pneumonia in frail geriatric patients and is a major cause of mortality, due to the colonisation of microbes in vulnerable patients. This type of pneumonia has been associated with an increase in respiratory pathogens in the oral cavity. The aim of this study was to evaluate the effects of chlorhexidine compared to routine oral care in edentulous geriatric inpatients.

METHODS A double-blind, parallel-group randomised controlled trial was carried out. The intervention group received oral care with chlorhexidine $0.2 \%$, while the control group received routine oral care with thymol. Nurses provided oral care with assigned solutions of $20 \mathrm{~mL}$ once daily over seven days. Oral cavity assessment using the Brief Oral Health Status Examination form was performed before each oral care procedure. Data on medication received and the subsequent development of aspiration pneumonia was recorded. An oral swab was performed on Day 7 to obtain specimens to test for colonisation.

RESULTS The final sample consisted of 35 (control) and 43 (intervention) patients. Chlorhexidine was effective in reducing oral colonisation compared to routine oral care with thymol $(p<0.001)$. The risk of oral bacterial colonisation was nearly three times higher in the thymol group compared to the chlorhexidine group.

CONCLUSION The use of chlorhexidine $0.2 \%$ significantly reduced oral colonisation and is recommended as an easier and more cost-effective alternative for oral hygiene.
\end{abstract}

Keywords: aspiration, chlorhexidine, geriatrics, oral, pneumonia

\section{INTRODUCTION}

It has been widely reported that oral colonisation, which results from poor oral care, is one of the factors that cause aspiration pneumonia in the geriatric population. ${ }^{(1-3)}$ Ineffective oral care can cause microbes to colonise the oral cavity. An increase in respiratory pathogens in the oral cavity has been reported to heighten the risk of aspiration pneumonia in geriatric patients..$^{(4,5)}$

Chlorhexidine has been found to be an effective agent in reducing oral colonisation and hence the incidence of pneumonia. ${ }^{(6-8)}$ Its clinical effectiveness in reducing oral colonisation has been well-proven among mechanically ventilated patients. ${ }^{(3,9)}$ In a systematic review conducted by Lam et al, 15 studies utilised chlorhexidine as part of an oral healthcare protocol. ${ }^{(10)}$ Subject groups included in these studies were patients admitted to the intensive care unit, those undergoing cancer therapy and institutionalised patients with cerebral palsy. Although the benefits of chlorhexidine have been widely established, there is limited research on its efficacy in the geriatric population. ${ }^{(11,12)}$

The World Health Organization (WHO) stated that there is a need to extend research on oral care for the elderly due to an increasing global incidence of non-communicable diseases. ${ }^{(11,12)}$ A worldwide survey by the $\mathrm{WHO}$ on the global oral health of older people revealed that oral health programmes for the elderly are very rare, reflecting the lack of oral health policies. ${ }^{(13)}$ The WHO highly recommended that countries establish oral health programmes to meet the needs of the elderly. It emphasised the need to define relevant and measurable goals toward selecting a suitable intervention, in order to improve the oral health of the elderly and eventually prevent noncommunicable diseases. ${ }^{(13)}$ The present study also aimed to address the recommendation for managing pneumonia from the United States Centers for Disease Control and Prevention. ${ }^{(14)}$ In this clinical practice guideline, the efficacy of chlorhexidine is still an unresolved issue, indicating insufficient evidence or no existing consensus on its efficacy. ${ }^{(14)}$ These research gaps need to be addressed to achieve the recommendations of the WHO. Therefore, this study sought to provide evidence of the efficacy of chlorhexidine in reducing oral colonisation in the geriatric population. It is hoped that the findings can benefit health organisations, the nursing profession and patients.

The current practice in the setting of the present study, a tertiary teaching hospital, is the use of thymol gargle. Based on observations, this method was not effective in reducing oral colonisation; hence, this study aimed to examine the use of chlorhexidine as an alternative oral care intervention in reducing colonisation of oral pathogens among geriatric patients. The specific objectives were to: (a) determine the baseline oral colonisation mean index score in both the intervention (chlorhexidine) and control (routine oral care solution) groups; (b) compare the oral colonisation mean index score between those exposed to chlorhexidine and to routine oral care solution;

\footnotetext{
${ }^{1}$ Department of Nursing Science, Faculty of Medicine, University of Malaya, Kuala Lumpur, ${ }^{2}$ Department of Nursing, Faculty of Health Science, Universiti Teknologi MARA, Puncak Alam Campus, Selangor, ${ }^{3}$ Department of Medicine, Faculty of Medicine, ${ }^{4}$ Department of Biomedical Imaging \& University of Malaya Research Imaging Centre, University of Malaya, Kuala Lumpur, Malaysia

Correspondence: Dr Mei Chan Chong, Lecturer, Department of Nursing Science, Faculty of Medicine, University of Malaya, 50603 Kuala Lumpur, Malaysia. mcchong@um.edu.my
} 
(c) examine the difference in incidence rates of aspiration pneumonia during hospitalisation in both groups; and (d) examine the oral health condition in both groups using the Kayser-Jones Brief Oral Health Status Examination (BOHSE) score tool. The alternative hypotheses were: no difference in bacteria colonisation between the intervention and routine oral care groups; and no difference in the incidence rate of aspiration pneumonia between the intervention and routine oral care groups.

\section{METHODS}

The study was a randomised clinical trial conducted in the geriatric ward of University Malaya Medical Centre, Kuala Lumpur, Malaysia. The study was registered with the Australian New Zealand Clinical Trials Registry (Registration ID ACTRN12614001259639) and approval was obtained from the hospital ethics committee prior to the study. Equal randomisation was used at a ratio of 1:1 for the parallel groups.

The sample size was calculated using the formula by Sakpal. ${ }^{(15)}$ The mean difference of $0.5 \pm 0.71$ was calculated based on the results of previous studies by Clavero et al. ${ }^{(16)}$ In order to reduce the risk of committing a Type II error due to insufficient sample size and statistical power, the power was fixed at 0.80 with an alpha value of 0.05 . To compensate for potential attrition of subjects during the course of the study, $10 \%$ more subjects were recruited above the estimated sample size; the total sample size was 90 (45 in each group). Eligible participants were patients aged $\geq 65$ years who had been admitted to the geriatric unit, were edentulous with or without dentures, and had functional impairment. Those who were excluded had current oral infections or a diagnosis of pneumonia during admission. Patients also had to ensure that they were not on antibiotics for at least two weeks before enrolling in the study.

After informed consent was obtained from family members or caregivers, patients were randomised to one of two arms using block randomisation (1:1) with a random number table. All random numbers were produced by the Stat Trek Random Number Generator. The researcher developed a list of numbers from 1 to 300; from the list, 90 participants were randomly chosen from a random number table. Patients eligible for the intervention and control groups were assigned a number from 1 to 90 . Participants assigned to the first column of numbers received chlorhexidine $0.2 \%$ mouthwash as Bottle A (i.e. intervention group), while those assigned to the second column of numbers received thymol gargle as Bottle B (i.e. control group). Both solutions were provided by the hospital pharmacy. Both bottles were of similar appearance in bottle type and size, amount of solution, and colour. They were labelled A or B as a code key. Patients, nurses and medical laboratory technicians were not aware of the code key, as this was a double-blind study.

All participants received oral care from nurses with their assigned solution for seven days ( $20 \mathrm{~mL}$ once daily). Nurses used an oral care checklist developed by the researcher, based on a previous study, ${ }^{(17)}$ consisting of 20 required steps for the oral care of geriatric patients. The researcher demonstrated the procedure to the nurses using the checklist and the nurses were asked to repeat the procedure. The researcher continued to monitor the oral care of patients to ensure adherence to the checklist. Prior to each oral care session, an oral cavity assessment was performed using the BOHSE form. The nurses examined patients' records for medication received and development of aspiration pneumonia from Day 1-7 of the study. On Day 1, after oral assessment and oral care procedure, oral swabs were taken as baseline data. Oral cavity assessment was performed throughout the study period to identify any side effects of the assigned solution. On Day 7, after the oral care procedure, oral swabs were taken from the buccal, occlusal and lingual sides for culture and sensitivity, in order to determine the colonisation of microorganisms. The intervention and control groups underwent the same oral care procedure except for the solutions used.

The effectiveness of the intervention was evaluated using the BOHSE, as well as oral swab sampling and laboratory testing. Permission to use BOHSE was granted by a representative of the author. BOHSE was designed to evaluate the oral condition of geriatric patients with or without cognitive impairment and has been validated in the American population; its test-retest reliability $(r=0.79-0.83)$, interrater reliability $(r=0.40-0.68)$ and content validity have been established as statistically significant by six field experts. ${ }^{(18)}$ In order to suit the Malaysian context, the content validity of this study was established by six field experts, namely two nurse managers, two consultant geriatricians and two nursing lecturers, all of whom supported the relevance of the BOHSE oral cavity assessment tool. The tool also received a test-retest reliability score of $r=-0.90$.

SPSS Statistics version 20.0 (IBM Corp, Armonk, NY, USA) was used for data analysis. Demographic, clinical and microbiology data was examined using chi-square test and independent $t$-test. Chi-square test was used to examine the effectiveness of chlorhexidine. Repeated measures analysis of variance was used to test the oral health condition of both groups. Statistical significance was set at $p$-value $<0.05$.

\section{RESULTS}

Daily screenings of all eligible geriatric patients admitted to the ward were conducted from 5 February 2013 to 31 March 2013. A total of 90 patients who fulfilled the criteria were recruited. Fig. 1 shows the patient recruitment procedure. Patients matching the inclusion criteria were randomised into two groups of 45 subjects each. Out of the 90 patients selected, 78 patients were available for final analysis. Six patients were lost to follow-up due to discharge from the ward, two patients refused to continue and four patients died before completion of the intervention.

Table I shows the demographic and clinical characteristics of patients in the study. The mean age of patients in the intervention (chlorhexidine) and routine oral care (thymol) groups was 81.2 years and 79.9 years, respectively. There was no significant difference in demographic, clinical or microbiology characteristics between the two groups, suggesting that they were homogenous and hence comparable.

Oral colonisation at baseline and postoral care was compared between the intervention and control groups, as shown in 


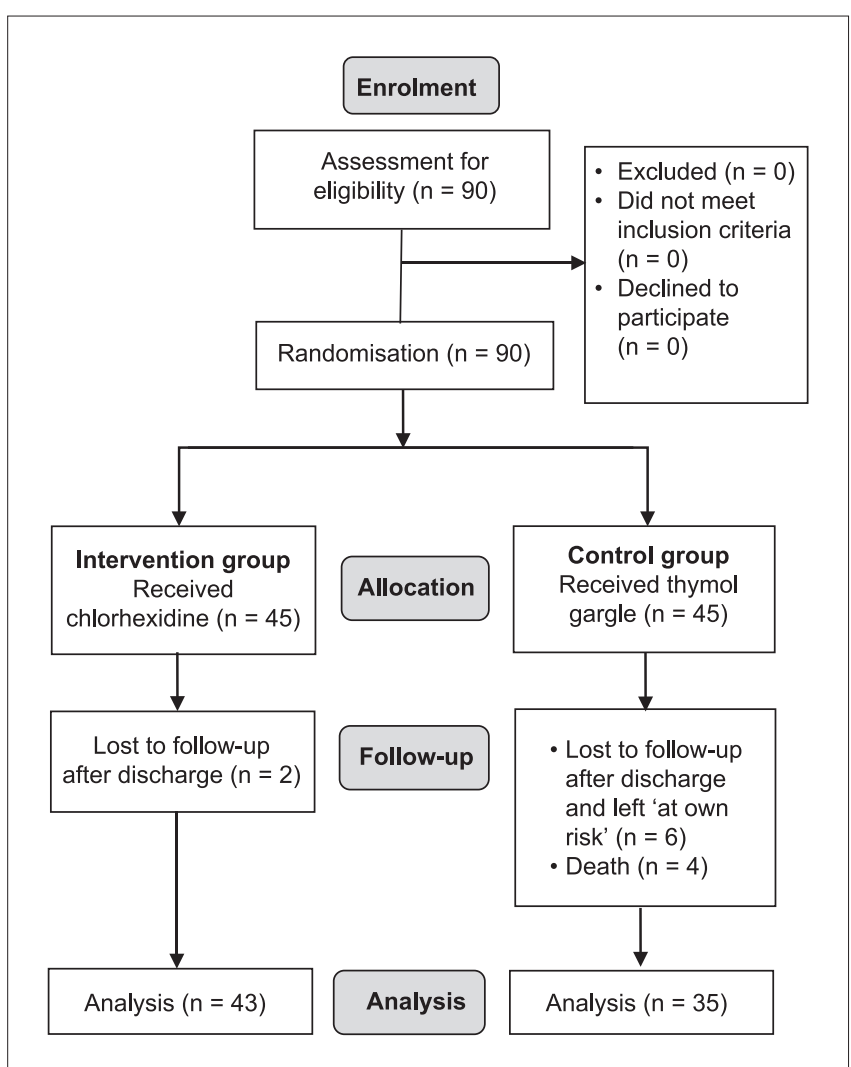

Fig. 1 Flowchart shows the participant enrolment, treatment allocation and follow-up process.

Table II. The microbial count from the oral swabs was measured in colony-forming units $(\mathrm{CFU} / \mathrm{mL})$ to determine oral colonisation. No significant difference was found in oral colonisation between the groups at baseline $(p=0.317)$. About half of the patients harboured more than 200 colonies.

In the chlorhexidine group, the oral colony count was significantly reduced after oral care compared to the baseline count $(p<0.001)$. About $32(74.4 \%)$ patients from the chlorhexidine group showed reduction in oral colonisation from the baseline results. In the control group, there was no significant reduction in oral colonisation after oral care with thymol. Approximately $54.3 \%$ of patients in the control group harboured more than 200 colonies, $20.0 \%$ had $50-200$ colonies and $25.7 \%$ of patients had less than 50 colonies. Hence, there was a significant difference in oral colonisation between the two groups $(p<0.001)$ from baseline to postoral care. Only 9 (25.7\%) patients in the control group showed a reduction in oral colonisation from baseline to postoral care. The results also revealed that oral colonisation in the control group did not differ significantly at baseline and postoral care $(p=0.375)$. The control group, who received routine oral care solutions, had nearly a three-fold risk of developing oral bacterial colonisation compared to the intervention group (95\% confidence interval 1.61, 5.22).

Results of BOHSE oral cavity assessment showed no significant difference in baseline BOHSE score between the $\mathrm{CHX}$ group and the routine oral care group $(1.53 \pm 1.29$ vs. $1.77 \pm 1.30 ; p=0.427)$ (Table III). BOHSE results after intervention also did not show any significant difference between the two groups $(p=0.165)$. No
Table I. Demographic and clinical characteristics of participants $(n=78)$.

\begin{tabular}{|c|c|c|c|c|}
\hline \multirow[t]{2}{*}{ Characteristic } & \multicolumn{2}{|c|}{ No. (\%) } & \multirow[t]{2}{*}{ Stat (df) } & \multirow[t]{2}{*}{ p-value } \\
\hline & $\begin{array}{c}\text { CHX } \\
(n=43)\end{array}$ & $\begin{array}{l}\text { Thymol } \\
(n=35)\end{array}$ & & \\
\hline Age (yr) & $81.2 \pm 9.1^{*}$ & $79.9 \pm 6.6^{*}$ & $0.64(76)^{+}$ & 0.142 \\
\hline Gender & & & $0.26(1)^{\ddagger}$ & 0.612 \\
\hline Male & $16(37.2)$ & $15(42.9)$ & & \\
\hline Female & $27(62.8)$ & $20(57.1)$ & & \\
\hline Race & & & $1.04(2)^{\ddagger}$ & 0.594 \\
\hline Malay & $12(27.9)$ & $9(25.7)$ & & \\
\hline Chinese & $26(60.5)$ & $19(54.3)$ & & \\
\hline Indian & 5 (11.6) & $7(20.0)$ & & \\
\hline $\begin{array}{l}\text { Source of } \\
\text { admission }\end{array}$ & & & $0.55(2)^{\ddagger}$ & 0.761 \\
\hline Other ward & 7 (16.3) & $5(14.3)$ & & \\
\hline Home & $29(67.4)$ & $22(62.9)$ & & \\
\hline Nursing home & 7 (16.3) & $8(22.9)$ & & \\
\hline $\begin{array}{l}\text { Length of stay } \\
\text { (day) }\end{array}$ & $14.5 \pm 14.4^{*}$ & $14.1 \pm 7.1^{*}$ & $0.15(76)^{+}$ & 0.061 \\
\hline $\begin{array}{l}\text { Baseline oral } \\
\text { cavity score }\end{array}$ & $1.5 \pm 1.3^{*}$ & $1.7 \pm 1.3^{*}$ & $0.79(76)^{+}$ & 0.427 \\
\hline $\begin{array}{l}\text { Baseline swab } \\
\text { (CFU/mL) }\end{array}$ & & & $2.30(2)^{*}$ & 0.317 \\
\hline$<50$ & 7 (16.3) & $5(14.3)$ & & \\
\hline $50-200$ & $16(37.2)$ & $8(22.9)$ & & \\
\hline$>200$ & $20(46.5)$ & $22(62.9)$ & & \\
\hline
\end{tabular}

* Data presented as mean \pm standard deviation. Statistical analysis performed

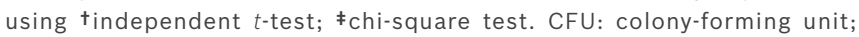
$\mathrm{CHX}$ : chlorhexidine group; df: degrees of freedom; thymol: thymol gargle group

Table II. Baseline and postoral colonisation by group.

\begin{tabular}{|c|c|c|c|c|}
\hline \multirow{2}{*}{$\begin{array}{l}\text { Oral } \\
\text { colonisation* } \\
\text { (CFU/mL) }\end{array}$} & \multicolumn{2}{|c|}{ No. (\%) } & \multirow{2}{*}{$\begin{array}{c}\text { chi-square } \\
\text { (df) }\end{array}$} & \multirow[t]{2}{*}{ p-value } \\
\hline & $\begin{array}{c}\text { CHX } \\
(n=43)\end{array}$ & $\begin{array}{l}\text { Thymol } \\
(n=35)\end{array}$ & & \\
\hline Baseline & & & $2.30(2)$ & 0.317 \\
\hline$<50$ & $7(16.3)$ & 5 (14.3) & & \\
\hline $50-200$ & $16(37.2)$ & $8(22.9)$ & & \\
\hline$>200$ & $20(46.5)$ & $22(62.9)$ & & \\
\hline Postoral care & & & $20.54(2)$ & $<0.001$ \\
\hline$<50$ & $32(74.4)$ & $9(25.7)$ & & \\
\hline $50-200$ & $6(14.0)$ & $7(20.0)$ & & \\
\hline$>200$ & 5 (11.6) & $19(54.3)$ & & \\
\hline
\end{tabular}

*Data is log-transformed, risk ratio $=2.9$. CFU: colony-forming unit; $\mathrm{CHX}$ : chlorhexidine group; df: degrees of freedom; thymol: thymol gargle group

Table III. Brief Oral Health Status Examination (BOHSE) scores in oral cavity assessments by group.

\begin{tabular}{|c|c|c|c|c|}
\hline \multirow{2}{*}{$\begin{array}{l}\text { BOHSE } \\
\text { score }\end{array}$} & \multicolumn{2}{|c|}{ Mean \pm standard deviation } & \multirow{2}{*}{$\begin{array}{l}t \text {-test } \\
\text { (df) }\end{array}$} & \multirow[t]{2}{*}{ p-value } \\
\hline & CHX & Thymol & & \\
\hline Baseline & $1.53 \pm 1.29$ & $1.77 \pm 1.30$ & $0.79(76)$ & 0.427 \\
\hline Postoral care & $1.25 \pm 1.21$ & $1.65 \pm 1.30$ & $1.40(76)$ & 0.165 \\
\hline
\end{tabular}

$\mathrm{CHX}$ : chlorhexidine group; df: degrees of freedom; thymol: thymol gargle group

side effects were observed in any participants during the study period, although two patients in the intervention group reported a bitter taste upon application. 


\section{DISCUSSION}

In the present study, both groups had high viable counts of oral microbial colonisation at baseline, which is consistent with other studies among the institutionalised elderly. ${ }^{(17,19,20)}$ The intervention group, who received oral care with chlorhexidine mouthwash for seven days, showed a significant reduction in oral colonisation compared to the control group $(p<0.001)$. The null hypothesis of the study was rejected, as there was a beneficial and statistically significant effect from oral care with chlorhexidine mouthwash in reducing oral colonisation in these geriatric patients. The risk of oral bacterial colonisation was nearly three times higher in the control group compared to the intervention group.

These results are supported by previous research on the efficacy of chlorhexidine in reducing oral colonisation. A previous study conducted among the dependent elderly in Japan found that wiping oral mucosa with a sponge brush soaked in chlorhexidine decreased opportunistic infections in the pharyngeal region $(p<0.05) .{ }^{(14)}$ Another consistent finding from a study in Brazil was that chlorhexidine-based treatment had the ability to remove oral biofilm in 60 geriatric patients. ${ }^{(20)}$

In addition, the present study confirmed that daily application of chlorhexidine $0.2 \%$ solution is effective in reducing microbes in the oral cavity. The usual mouthwash regime includes two applications daily, but a once-daily application of chlorhexidine was implemented in this study, as it could reduce the treatment cost and even the complications of poor oral hygiene such as gingival inflammation. ${ }^{(16)}$ The use of chlorhexidine mouthwash should be incorporated in future routine oral care among elderly patients.

However, a firm conclusion could not be reached on the role of oral hygiene with chlorhexidine in preventing aspiration pneumonia in geriatric patients. A similar study in Japan was also unable to provide data on the incidence of aspiration pneumonia. ${ }^{(14)}$ The absence of aspiration pneumonia in our patients may have been due to the short-term effects of oral hyigene care over seven days and the small sample size. Previous research recommended having a study with a longer duration and higher number of patients, to possibly support the theory that effective oral care is an important contributing factor in the prevention of aspiration pneumonia. ${ }^{(7,21)}$

Patients in both the chlorhexidine and control groups did not show any significant differences $(p=0.165)$ in adverse effects or improvements to their oral cavity health. Even though it was not statistically significant, there was a slight reduction in the BOHSE scores of the intervention group from Day 3 compared to the control group, from a mean of 1.53 at baseline to 1.25. The reduction was due to changes in the formation of tongue coating in the intervention group. This finding suggests that chlorhexidine may improve oral cavity health, but further investigation with a larger sample and longer duration of study is needed.

Furthermore, the BOHSE method of measuring oral cavity health may not be suitable for older persons who are more dependent and edentulous. BOHSE scores were very low or not significant even in patients with higher oral colony growth (> 200 colonies) and, hence, poor oral hygiene, as the score is mainly used to assess dental hygiene and risk of developing caries lesions in subjects with teeth. ${ }^{(18)}$ This highlights the need to develop an assessment tool that would be more applicable to the edentulous elderly.

The present study helped to create awareness among healthcare providers, especially nurses, that chlorhexidine mouthwash is effective in reducing oral colonisation. It also provided nurses and caregivers with knowledge in providing oral care to the elderly. Nurses suggesting the use of chlorhexidine in daily oral care practice could be a cost-effective way of preventing complications related to colonisation in the oral cavity by harmful microorganisms.

The strength of this study was the randomisation procedure used to allocate the two types of solutions, which eliminated bias and confounders, as well as successful blinding in mouthwash preparation. The study may also serve as a reference for the similar use of chlorhexidine in other settings and populations to reduce the rate of oral infection and aspiration pneumonia. The main finding of this study corresponds with that from previous research on the efficacy of chlorhexidine in reducing oral colonisation among vulnerable older patients. One limitation was the inability to perform advanced microbiology examinations due to insufficient funding. Although chlorhexidine mouthwash was effective in reducing the viable count of oral pathogens in the intervention group, identification of microorganisms was not performed. This will be considered in future studies. Another factor that was not assessed was denture hygiene. Although all patients with removable dentures were instructed to remove them before oral care, procedures for denture care were not observed. Contamination of dentures could increase oral colonisation and, hence, the incidence of aspiration pneumonia among geriatric patients. ${ }^{(22)} \mathrm{A}$ recent study in Brazil found that immersion of dentures in chlorhexidine solution was able to remove denture biofilm; this was suggested as an auxiliary method for cleaning dentures. ${ }^{(20)}$ This factor should be considered in future research. The challenges in undertaking the study were cost (as equipment, solutions and laboratory equipment required a significant amount of funding) and the need to train and supervise research assistants or healthcare workers to ensure that procedures were performed correctly.

Findings from this study can increase awareness among healthcare providers and patient caregivers on the importance of practising oral care with the recommended solution. It also provides strong clinical evidence to encourage stakeholders of organisations to consider prescribing chlorhexidine to patients for oral care although it is more expensive than thymol solution. In the long-term, the use of chlorhexidine may reduce the patient's length of stay in hospital and hospitalisation costs, and improve quality of life for patients and caregivers. ${ }^{(21)}$

Usage of antibiotics and other medical care equipment can be reduced through the use of chlorhexidine; in previous research involving chlorhexidine, it was reported that the mean cost per patient was ten times less than the cost of antibiotics needed. ${ }^{(23)}$ Similarly, chlorhexidine was found to be a safe, cost-effective intervention for ventilator-associated pneumonia without side effects. $^{(24)}$

In view of the limitations of the study, it should be replicated by other hospitals for a longer period of time to substantiate findings, with the inclusion of advanced microbiology examinations to 
identify the types of microorganisms. Such research would yield more reliable evidence to address the reduction of aspiration pneumonia and provide data to support policy change within healthcare organisations, such as developing standard procedures for oral care in the clinical setting. Future research should also explore the long-term advantages of chlorhexidine usage in reducing aspiration pneumonia, which has the potential to reduce hospital stay, improve patient outcome and lead to cost savings.

In conclusion, the present study showed that the use of chlorhexidine in the oral care of edentulous geriatric patients has a positive effect in reducing oral colonisation when compared to routine oral care. The higher count of oral microbial colonisation among the study subjects highlights the importance of establishing standardised strategies in oral hygiene. These strategies can be enhanced through the use of an appropriate mouthwash (i.e. a single application of chlorhexidine $0.2 \%$ ), which should be maintained in order to preserve its benefits over time. This could reduce the cost of treatment for oral complications resulting from poor hygiene and would be easy to incorporate into the routine oral care of frail geriatric patients.

\section{REFERENCES}

1. Komulainen $K$, Ylöstalo $P$, Syrjälä $A M$, et al. Determinants for preventive oral health care need among community-dwelling older people: a population-based study. Spec Care Dentist 2014; 34:19-26.

2. Stewart SL. Daily oral hygiene in residential care. Can J Dent Hyg 2013; 47:25-30.

3. Weening-Verbree L, Huisman-de Waal G, van Dusseldorp L, van Achterberg T, Schoonhoven L. Oral health care in older people in long term care facilities: a systematic review of implementation strategies. Int J Nurs Stud 2013; 50:569-82.

4. Kim JK, Baker LA, Davarian S, Crimmins E. Oral health problems and mortality. J Dent Sci 2013; 8:115-20.

5. van der Maarel-Wierink CD, Vanobbergen JN, Bronkhorst EM, Schols JM, de Baat C. Meta-analysis of dysphagia and aspiration pneumonia in frail elders. J Dent Res 2011; 90:1398-404.

6. Kusahara DM. Peterlini MA, Pedreira ML. Oral care with $0.12 \%$ chlorhexidine for the prevention of ventilator-associated pneumonia in critically ill children: randomised, controlled and double blind trial. Int J Nurs Stud 2012; 49:1354-63.

7. van der Maarel-Wierink CD, Vanobbergen JN, Bronkhorst EM, Schols JM, de Baat C. Oral health care and aspiration pneunomia in frail older people: a systematic literature review. Gerodontology 2013; 30:3-9.

8. Sharma SK, Kaur J. Randomized control trial on efficacy of chlorhexidine mouth care in prevention of Ventilator Associated Pneumonia (VAP). Nurs Midwifery Res J 2012; 8:169-78.

9. Hoshijima $\mathrm{H}$, Kuratani $\mathrm{N}$, Takeuchi $\mathrm{R}$, et al. Effects of oral hygiene using chlorhexidine on preventing ventilator-associated pneumonia in criticalcare settings: A meta-analysis of randomized controlled trials. J Dent Sci 2013; 8:348-57.

10. Lam OL, McGrath C, Li LS, Samaranayake LP. Effectiveness of oral hygiene interventions against oral and oropharyngeal reservoirs of aerobic and facultatively anaerobic gram-negative bacilli. Am J Infect Control 2012; 40:175-82.

11. Peterson, PE, Ogawa H. Strengthening the prevention of periodontal disease: the WHO approach. J Periodontol 2005; 76:2187-93.

12. Petersen PE, Kandelman D, Arpin S, Ogawa H. Global oral health of older people--call for public health action. Community Dent Health 2010; 27(4 Suppl 2):257-67.

13. Coffin SE, Klompas M, Classen D, et al. Strategies to prevent ventilatorassociated pneumonia in acute care hospitals. Infect Control Hosp Epidemiol 2008; 29 Suppl 1:S31-40.

14. Tashiro K, Katoh T, Yoshinari N, et al. The short-term effects of various oral care methods in dependent elderly: comparison between toothbrushing, tongue cleaning with sponge brush and wiping on oral mucous membrane by chlorhexidine. Gerodontology 2012; 29:e870-82.

15. Sakpal TV. Sample size estimation in clinical trial. Perspect Clin Res 2010; 1:67-9.

16. Clavero J, Baca P, Junco P, González MP. Effect of $0.2 \%$ chlorhexidine spray applied once or twice daily on plaque accumulation and gingival inflammation in a geriatric population. J Clin Periodontol 2003; 30:773-7.

17. Wårdh IM, Wikström MB. Long-term effects of using oral care aides at a nursing home for elderly dependent residents--a pilot study. Spec Care Dentist 2014; 34:64-9

18. Taub LFM. Oral health assessment of older adults: The Kayser-Jones Brief Oral Health Status Examination (BOHSE). In: The Harford Institute for Geriatric Nursing, New York University College of Nursing [online]. Available at: https://consultgeri.org/try-this/general-assessment/issue-18. pdf. Accessed September 30, 2012.

19. Yakiwchuk C, Bertone M, Ghiabi E, et al. Suction toothbrush use for dependent adults with dysphagia: A pilot examiner blind randomized clinical trial. Can J Dent Hyg 2013; 47:15-23.

20. de Andrade IM, Cruz PC, Silva-Lovato $\mathrm{CH}$, et al. Effect of chlorhexidine on denture biofilm accumulation. J Prosthodont 2012; 21:2-6.

21. Tada A, Miura H. Prevention of aspiration pneumonia (AP) with oral care. Arch Gerontol Geriatr 2012; 55:16-21.

22. Valentini F, Luz MS, Boscato N, Pereira-Cenci T. Biofilm formation on denture liners in a randomised controlled in situ trial. J Dent 2013; 41:420-7.

23. Tantipong $\mathrm{H}$, Morkchareonpong $\mathrm{C}$, Jaiyindee $\mathrm{S}$, Thamlikitkul V. Randomized controlled trial and meta-analysis of oral decontamination with $2 \%$ chlorhexidine solution for the prevention of ventilator-associated pneumonia. Infect Control Hosp Epidemiol 2008; 29:131-6.

24. Koeman M, van der Ven AJ, Hak E, et al. Oral decontamination with chlorhexidine reduces the incidence of ventilator-associated pneumonia. Am J Respir Crit Care Med 2006; 173:1348-55. 